\title{
Automatic Recognition and Verification of Handwritten Legal and Courtesy Amounts in English Language Present on Bank Cheques
}

\author{
Ajay K. Talele \\ Department of Electronics \\ Dr..B.A.T.U. Lonere.
}

\author{
Sanjay L Nalbalwar \\ Department of Electronics \\ Dr..B.A.T.U. Lonere
}

\author{
Milind E. Rane \\ Department of Electronics \\ V.I.T, Pune.
}

\begin{abstract}
The main objective of the work proposed here is to evolve with novel, effective, robust and reliable image processing techniques, which can clearly separate, recognize and verify the legal and courtesy amount written in English language present on bank cheques. To achieve this, a database of handwritten samples was initially built containing all the possible words which may appear in cheques from a number of users. Using the database the features extracted from it, an attempt has been made to recognize the legal and courtesy amounts present in the cheque and to crosscheck them.
\end{abstract}

\section{Keywords}

Bank cheques, OCR, legal and courtesy amount.

\section{INTRODUCTION}

Since the advent of digital computers machine simulation of human reading has become the subject of intensive research. The reason for such an effort was not only because of its challenge on simulation of human reading but also because it provides efficient applications for the automatic processing of bulk amount of papers i.e. transferring the data present on bank cheques, commercial forms, government records, credit card imprints, envelops into machine readable format. Document analysis or more precisely, document image analysis, is the process that performs the overall interpretation of document images. Thus document analysis is concerned with the global issues involved in recognition of written language in images. It adds to the Optical Character Recognition (OCR) a superstructure that establishes the organization of the document and applies outside knowledge in interpreting it. In practice then, a document analysis system performs the basic tasks of image segmentation, layout understanding, symbol recognition and application of contextual rules in an integrated manner. The current work in this area is mainly about analysis and recognition of form documents (different application forms, bank documents etc).

The latest work published in the area of hand written English bank cheque processing includes three main modules that allow for fully automated bank check processing [1]. Some of the already existing documents reading systems have very low recognition rates. A multi-layered corroboration-based cheque reading system in which, to control the error rate, corroborations at the character, digits level, at the word image level, between fields such as courtesy and legal amount, and with external sources was implemented[2]. The complete recognition performance with the automated field segmentation is estimated at $44 \%$ to $49 \%$. Another system known as A2iA system [3] was developed in France in 1996. The system recognizes both legal and courtesy amounts on bank cheques. The system has recognition rates between $50 \%$ and $65 \%$.

Even though many powerful and efficient techniques are available for the recognition of printed documents handwritten recognition is still an unsolved problem [4]. It is also the most difficult part of the area because depending on the style of the writer and the speed of writing some characters may vary in size or shape, in stroke number, and order dynamic variation. Human visual system is insensitive to the position orientation and size changes of characters. However, representation of the knowledge of these variations affects the recognition rates a great deal. The main objective of this work is to evolve with novel, effective, robust and reliable image processing techniques, which can clearly separate, recognize and verify the legal and courtesy amount written in English language present on bank cheques. The rest of paper is arranged as Section 2 gives system description, Section 3 describes the proposed algorithm, and Section 4 provides the results and discussion.

\section{SYSTEM DESCRIPTION}

The block diagram of system is given in figure 1. Initially, all the possible handwritten words and symbols that can occur in the legal and courtesy amounts have to be collected from the intended writer. This process is called the 'enrollment' phase. The main steps involved in this phase are preprocessing, feature extraction, and storage.

In the recognition phase the handwritten cheque is scanned using a digital scanner to get its image. The various fields are then extracted using the prior knowledge of the format of the cheque image. To recognize the handwritten information present on the cheque, the features of the hand written information are extracted and are compared with the already stored features

1) Enrollment:-

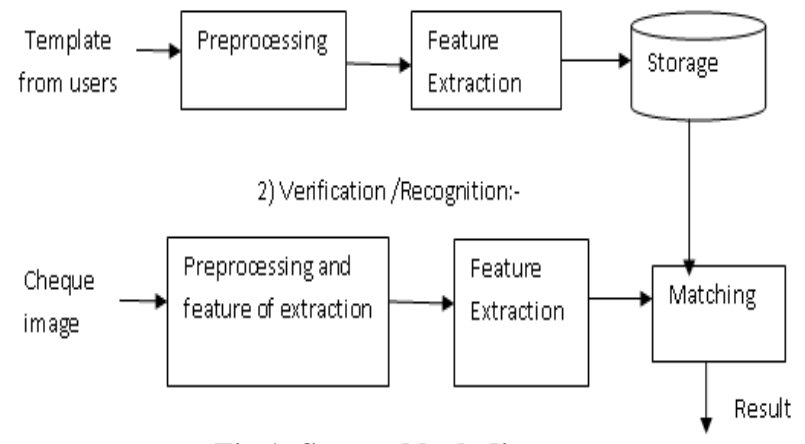

Fig 1: System block diagram 


\section{SOFTWARE DESCRIPTION}

The main steps in the algorithm are given as Enrollment and Recognition.

\subsection{Enrollment}

The different steps are preprocessing, Determination of coordinates of the lines and extraction of elements, and Feature extraction

Preprocessing: The main steps involved are:

Binarization: Binarization is done using thresholding with a manually defined threshold or Otsu's method.

Noise removal: Noise removal is done using median filter.

Skew elimination: Using Radon transform, the angle which gives maximum projection result is found out. Rotate the image in opposite direction with this angle.

\subsubsection{Determination of coordinates of the lines and extraction of elements:}

For every row, check the total number of black points, $n$. If $n$ is greater than threshold, detect it as a line.

Store the coordinate. Eliminate the case in which the width of a line is more than one pixel (thinning). Repeat the above mentioned steps for columns. Using the coordinates obtained in above steps; perform the cropping of image to get the database elements. The extracted words are as shown in Figure 2.

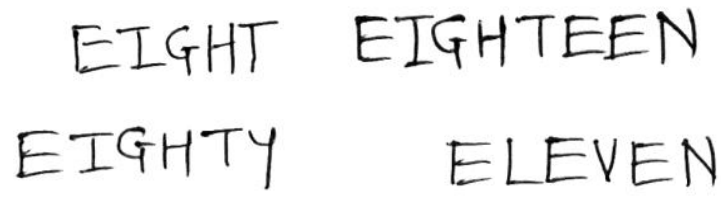

Fig 1: Sample extracted words

\subsubsection{Feature extraction:}

Geometric Moment features are used. $(p+q)$ th order moment is defined as,

$$
\boldsymbol{M}_{p q}=\sum_{x} \sum_{y} x^{p} y^{q} I(x, y)
$$

M00 is the area calculated as,

$$
\boldsymbol{M}_{\mathrm{OO}}=\sum_{x} \sum_{y} I(x, y)
$$

Centroid can be computed as,

$$
x^{\prime}=\frac{M_{10}}{M_{00}} \quad y^{\prime}=\frac{M_{01}}{M_{00}}
$$

Central moments given by,

$$
M_{p q}=\sum_{x} \sum_{y}\left(x-x^{\prime}\right)^{p}\left(y-y^{\prime}\right)^{q} I(x, y)
$$

where $\left(x^{\prime}, y^{\prime}\right)$ is centroid.

Important features of the words are its aspect ratio. Aspect ratio of a word image is the ratio of the width of the word to the height of the word. Ascenders and descenders in words is shown in Figure 3

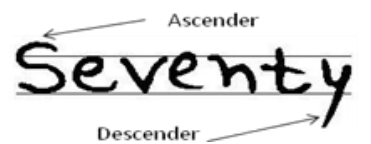

Fig 3: Ascenders and descenders in a word
After segmentation of all the digits in the courtesy amount, features are extracted from the digits. Three features are mainly used for all the numbers namely Cavity features, Profile features, Number and location of closed loops.

\subsubsection{Extraction of cavity features from the digit}

a. Locating the left and right cavities are shown in Figure 4.

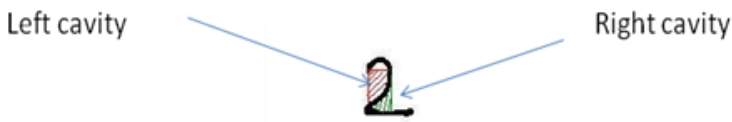

Fig 4: Left and right cavities

b. Locating the top and bottom cavities are shown in Figure 5. Top cavity

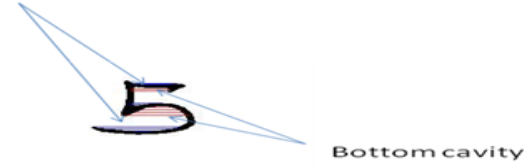

Fig 5 : Top and bottom cavities

\subsubsection{Profile features}

The left and right profiles of the digits are used as the feature vectors. These vectors are then matched using Dynamic Time Warping (DTW) algorithm. DTW holds an advantage over other matching techniques, in that the effect of variations in time eliminated. In short, the DTW algorithm matches the shapes of two vectors independent of the time variations. If the shapes are exactly similar, the output of the DTW algorithm is zero.

\subsubsection{Closed loops}

Number and location of closed loops in a digit serves as another feature as shown in Figure 6.

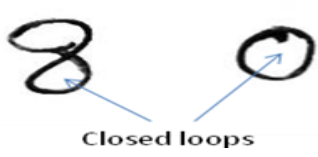

Fig 6 : Closed loops

From the presence or absence of closed loop, two major groups of digits can be made first group including $0,6,8,9$ which carry a closed loop and the second one including $1,2,3,5,7$. The digit ' 4 ' may or may not have a closed loop present depending upon the writing style.

\subsection{Preprocessing:}

Binarization, noise removal and skew elimination are performed on the cheque image. The processes cheque is a shown in Figure 7.

\subsubsection{Extraction of legal amount}

a) Baseline deletion: All the horizontal lines in the cheque which are of length greater than one fourth of the cheque length are determined. The top and bottom boundaries are excluded. Multiple counting of thick lines is then eliminated .The lines are then sorted as per length and their positions are determined. For all the lines the leftmost and the rightmost points are determined. The lines are then deleted from leftmost to rightmost point with an assumed thickness as shown in Figure 8 and Figure 9.

b) Reconstruction: A deleted line as discussed in 3.2(a) is then considered as reference lines. All the black pixels 


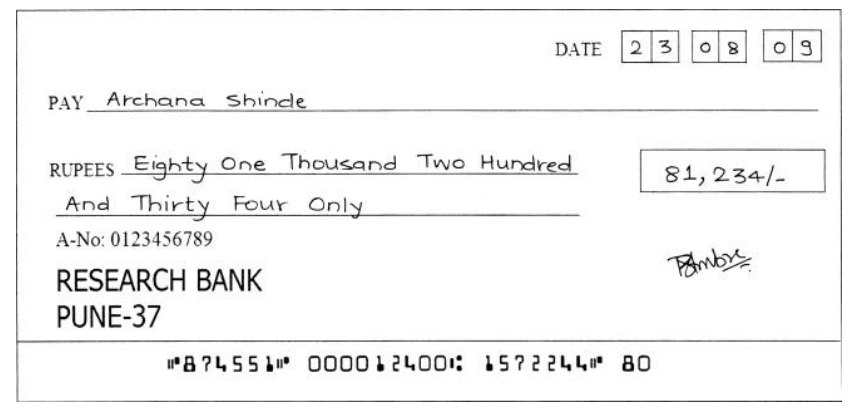

Fig 7 : Preprocessed cheque image

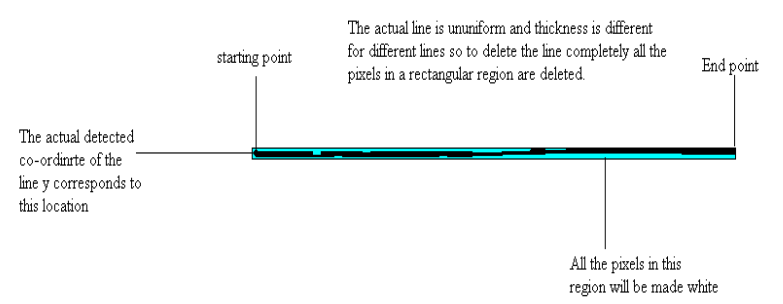

Fig 8 : Baseline elimination

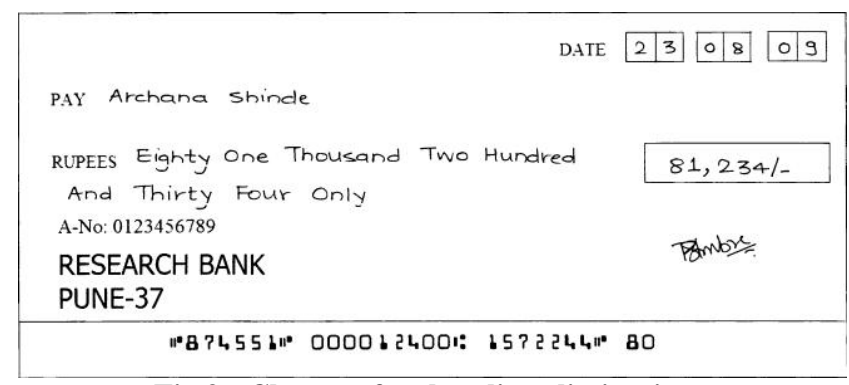

Fig 9 : Cheque after baseline elimination

immediately below the reference line are determined by scanning. Number of discontinuities and the leftmost and the rightmost pixel for every discontinuity that is immediately below the reference line are determined. Determine all the black pixels immediately above the reference line. For every discontinuity that is immediately below the reference line and the black pixels above the reference line part nearest to the leftmost and rightmost reference line are found out. Then the pair with shortest distance out of these two is found out. The selected pair is joined by assuming linear segment between the points. Repeat the procedure for other reference lines.

Changing the matching point for smooth line reconstruction. Here the matching point is moved left or right to get the corner black point as matching point. The thickness is taken to be the minimum of the thicknesses above and below the reference lines. The thickness is taken to be the minimum of the thicknesses above and below the reference lines.

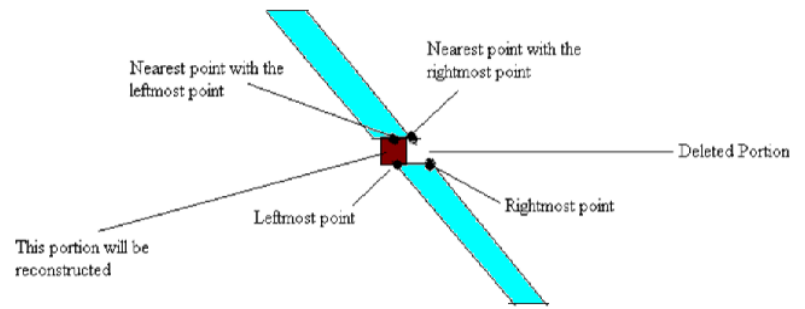

Fig 10 : Diagrammatic representation of the method

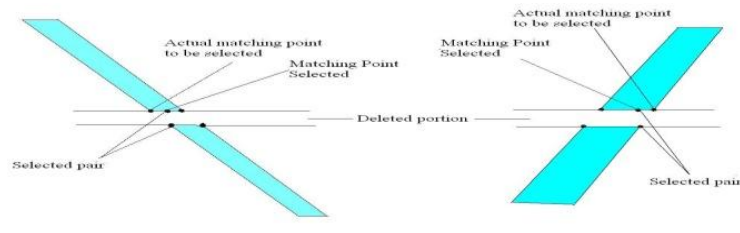

Fig 11 : Changing the matching point for smooth line reconstruction

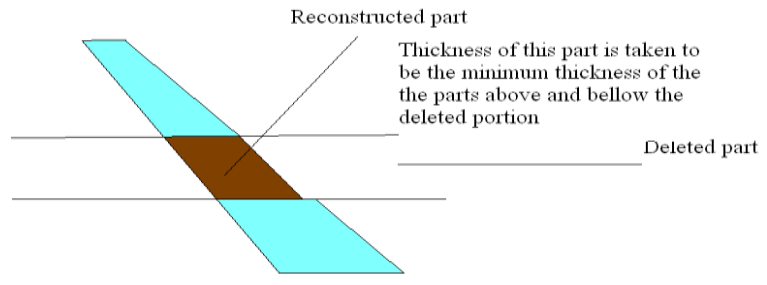

Fig 12 : Deciding the thickness of the reconstructed part

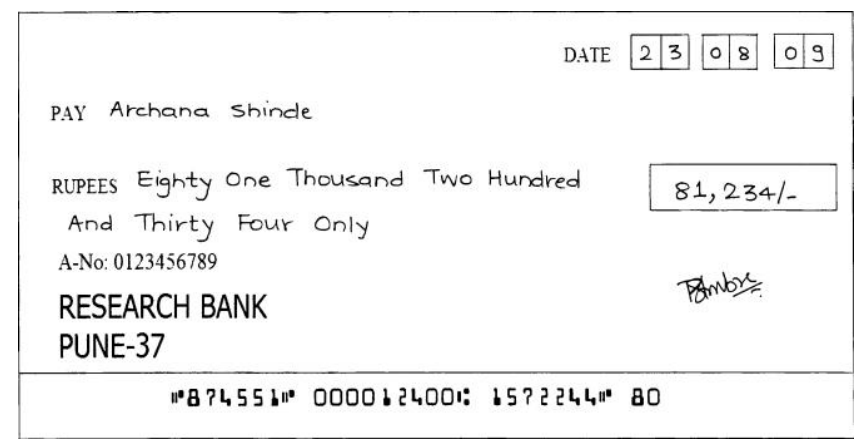

Fig 13 : Cheque image after reconstruction

c) Segmentation of words: Create a window of suitable dimensions and move it along the line. For every $\mathrm{x}-$ coordinate, store the number of black pixels in the window. If this count is less than the threshold, treat this $\mathrm{x}$-coordinate as a point in between the two words. If two consecutive windows satisfy the threshold condition, use first window to find the separation point. Using all the separation points, crop out the words as separate images.

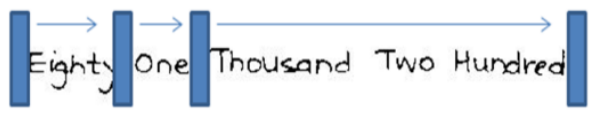

Fig 14 : Segmentation of words

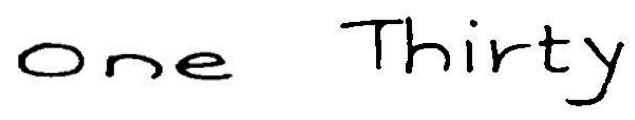

Fig 15 : Separated words

\subsubsection{Extraction of courtesy amount:}

The legal amount is cropped out from the cheque. Extraction of numbers is done using connected component labeling. Using connected component labeling each number is represented by a label.

Connected numbers are detected based on aspect ratio. Connected numbers are then separated using various methods as discussed in section 3.2.3. 


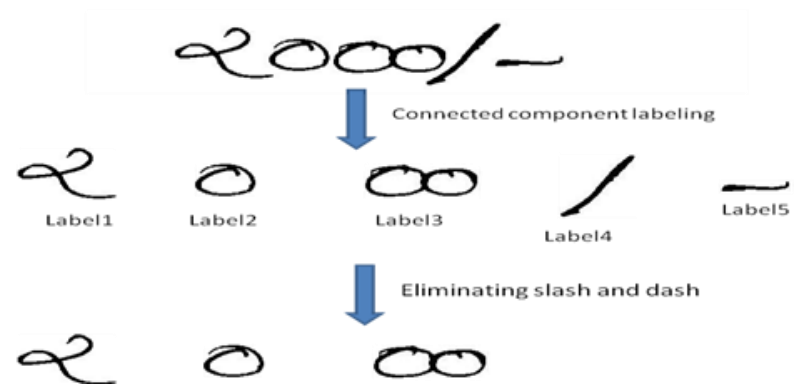

Fig 16 : Extraction of individual numbers

\subsubsection{Method for separation of connected numbers containing touching closed loops}

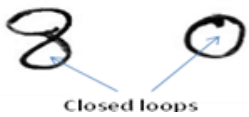

Fig 17 : Example of numbers containing closed loops Principle: When numbers containing closed loops are connected, the closed loop of one number touches that of other.
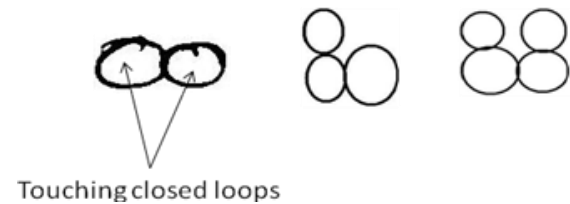

Fig 18 : Connected numbers with touching closed loops Methodology:

Step1: Find the number of closed loops in the joined numbers. Eliminate unnecessary closed loops. If number of closed loops equal to 1 use water reservoir model.

Step2: Number of closed loops $=2$

Check if the closed loops are touching

Case1: If line joining the centroids passes through the touching component then loops is touching

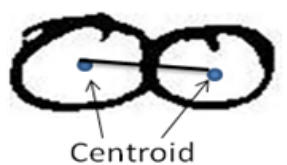

Fig 19 : Case 1

Case2: Line joining the centroids does not pass through the touching component as shown in Figure,

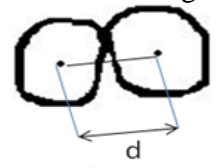

Fig 20 : Touching loops

If $\mathrm{d}<$ threshold then loops are touching.

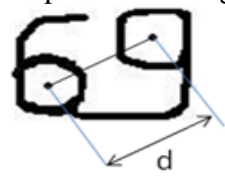

Fig 21 : Non touching loops

If $\mathrm{d}>$ threshold then loops are non-touching. Use water reservoir method.
Case3: To avoid false detection of the touching loops in number 8. Based on the difference between the $\mathrm{x}$ co-ordinates of centroids the case of no. 8 is eliminated.

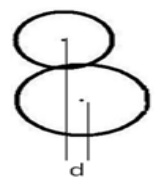

Fig 22 : Nonaligned centroids

Locate the initial point on the touching component
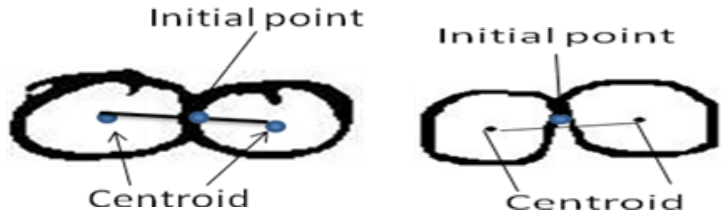

Fig 23 : Initial point location

Find the segmentation path along the midpoint of the touching component

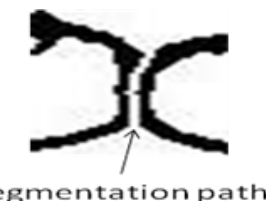

Fig 24 : Final segmentation path

Step 3: Closed loops $=3$ or 4. Consider a single pair of loops at a time. Follow the same procedure as for two loops.

Special cases: Intersecting loops.
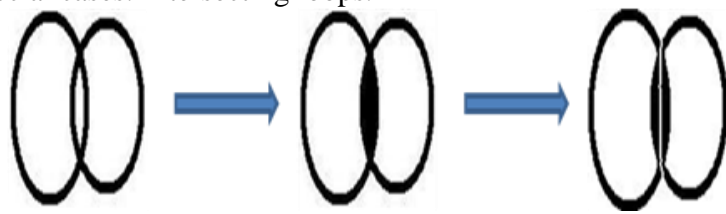

Fig 25 : Special case of intersecting loops

\subsubsection{Method for connected numbers not} containing touching closed loops

There is another model that is used to segment connected or touching components which do not have connected loops. This method is called water reservoir model.

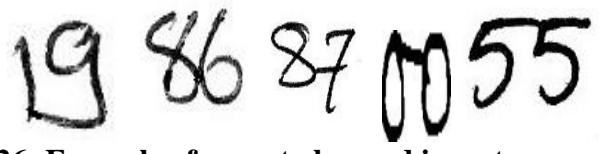

Fig 26: Example of cases to be used in water reservoir method

Definition of reservoir: It is the region formed after pouring the imaginary water from top and bottom after making the bounding box.

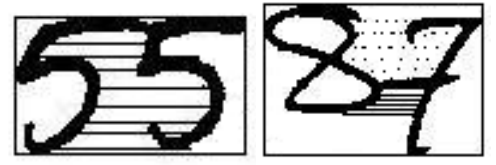

Fig 27 : Reservoirs formed

Need of reservoir model : Most of the times, touching position lies on the boundary of the best reservoirs. 


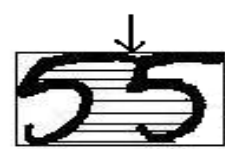

Fig 28 : Showing touching position of the component.

By best reservoir we mean the reservoir whose Centroid lies in $\mathrm{Vm}$ region. In order to find out this, we divide the image vertically and horizontally into 3 parts of length $25 \%, 50 \%$, $25 \%$. The $\mathrm{Vm}$ region is as shown below.

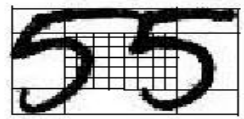

Fig 29 : Vm region

In some cases more than 1 reservoir's centroids lie in the Vm region.For example

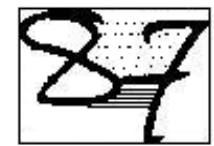

Fig 30 : Special case having 2 reservoirs' centroids in Vm region

Best reservoir is selected by finding the distance of centroids of all such reservoirs from two points which generally lie in the reservoir of touching component. It is found that $a / 3, b / 2$ and $2 \mathrm{a} / 3, \mathrm{~b} / 2$ are those points, where $\mathrm{a}$ and $\mathrm{b}$ are height and width of the image. The reservoir, whose centroid has minimum distance from these points, is selected.

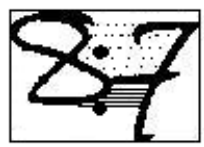

Fig 31 : Points to be considered for the above mentioned special case

There are two types of reservoirs viz. Top reservoir and Bottom reservoir. If reservoir is formed by pouring water from top (bottom), it is called as Top (Bottom) reservo

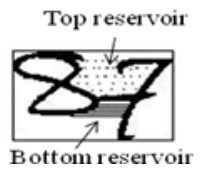

Fig 32 : Top and Bottom reservoirs

Methodology:

Finding the baseline

Baseline: Lowermost line in case of top reservoir and uppermost line in bottom reservoir

1. Find the starting point of operation on the boundary of best reservoir. The midpoint of the baseline is considered as the feature point.

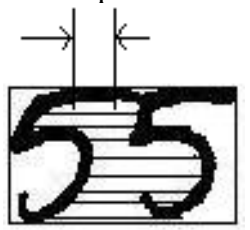

Fig 33 : Top Baseline of the reservoir

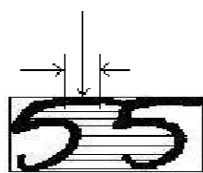

Fig 34 : Feature point shown by an arrow

2. Finding the node points

Node points can be found out by scanning leftwards and rightwards from feature point up till we get vertical rung more than $3 R / 2$ where $R$ is most occurring horizontal run.

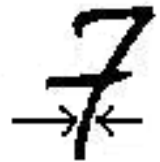

Fig 35 : Illustrating $\mathbf{R}$

From this we can get a sudden curve.

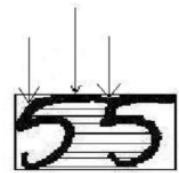

Fig 36 : Left node and right node on the two sides of feature point

3. Deleting unnecessary part.

Scanning from left node point to right node point, delete the columns which are having difference between first black pixel and last black pixel less than $3 R / 2$ where $R$ is defined previously.

Outputs:

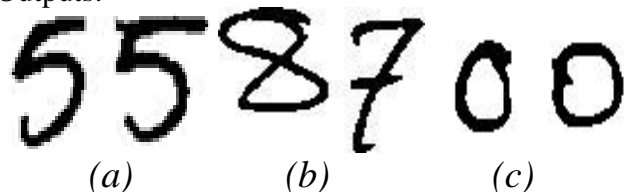

Fig 37 : Segmented images

\subsection{Number recognition:}

Cavity and closed loop features corresponding to each digit are mentioned below:

1. One (1): Very high aspect ratio (greater than 2.5). Aspect ratio is the ratio of width and height of the image. Presence of bottom cavity if written in style mentioned in Figure 38(b). Left cavity will be present if the horizontal bottom line is present.

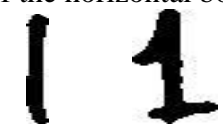

(a) (b)

Fig 38 : Styles of writing the digit ' 1 '

2. Two (2) one left cavity with centroid in upper region and one right cavity with centroid in lower region. Slope of the line joining the centroids of left and right cavities is always greater than 90 degrees. 


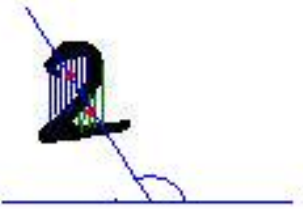

Fig 39 : Slope of line joining centroids in ' 2 '

3. Three (3) At least one left cavity and one right cavity with centroid in the central region.

4. Four (4) If no closed loop is there, a top cavity will be present. No. of cavities varies depending upon the slant of the vertical line. If divided in 9 equal parts, the no. of black pixels in bottom left region is very low (generally 0 ).

5. Five (5) one right cavity with centroid in upper region and one left cavity with centroid in lower region. Slope of the line joining the centroids of left and right cavities is always less than 110 degrees.

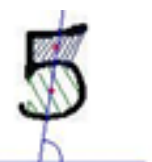

Fig 40 : Slope of line joining centroids in ' 5 '

6. Six (6) No left cavities. One right cavity and one closed loop located in the lower region.

7. Seven (7) At least one left cavity.

8. Eight (8) Two closed loops. One left and one right cavity with centroids in central region.

9. Nine (9) No right cavities and one closed loop located in the upper region.

10. Zero (0) No left, right, top and bottom cavities. If the image is divided into 9 regions of equal area, the central region has very less number of black pixels (generally ' 0 ').

It is highly impossible that other digits will have the similar feature. Single closed loop.

Along with these basic features, the left and right profiles of each digit are taken into account for recognition. If both left and right profiles match, the recognition process is over. If one of them doesn't match, the features mentioned above are used.

\subsection{Crosschecking of Legal and Courtesy amounts}

After obtaining the recognized courtesy amount, the probable words for this amount are found out. These words from the user database are then matched with the words written on the cheque. A verification decision is made depending upon the extent of similarity between the two. The matching is done using one-toone matching technique.

\section{RESULTS AND DISCUSSION}

We have proposed an offline system that can be used for different applications. A main aim is to recognize and verify the handwritten legal and courtesy amounts in English Language present on Indian bank cheques. The system takes an bank cheques and combination of different levels such as segmentation, recognition and post processing is made the system shows very efficient scheme of verification.

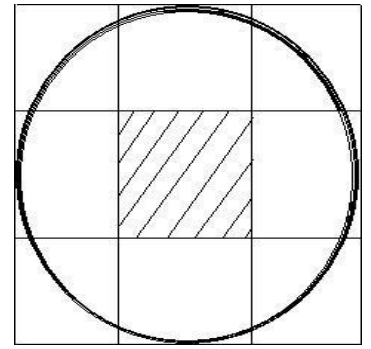

Fig 41 : Central region in ' 0 ' with no black pixels

The proposed system is tested on the database generated by taking the sample from the 50 different users. Each user writes cheques with different amount written on it. The algorithm segments effectively the handwritten legal and courtesy amounts on the cheque. The segmented data is recognized and verified for samples of 50 users and the results of the number recognition are given in Table1. The percentage probabilities of certain digit between 0-9 getting recognized as a digit in between 0-9 have been mentioned. Recognition rate observed is $92.59 \%$.

The last column "None" represents a state in which a system asks for manual recognition of digit. From the results, it can be observed that the digits containing closed loops i.e. 0,6,8,9 are recognized well as compared to the ones with no closed loops.

\section{REFERENCES}

[1] Rafael Palaciosa and Amar Gupta "A system for processing handwritten bank checks automatically", Image and Vision Computing ,Volume 26, Issue 10 pp.1297-1313, 2008.

[2] G.F. Houle, D-B. Aragon, R.W. Smith, M. Shridhar, and D. Kirnura ,"A multi-layered corroboration-based cheque reader". In Proc. of the International Association for Pattern Recognition Workshop on Document Andysis System, pp 495-546, Malvern, Pennsylvania USA, October 1996.

[3] S. Knerr, O. Baret, D. Price, and J.C. Simon. "The A2iA recognition system for handwritten cheques". In Proc. of the International Association for Pattern Recognition Workshop on Document Analysis Systems, pp. 431-494, Malvern, Pennsylvania USA, October 1996.

[4] Nafiz Arica \& Fatos T Yarman-Vural, "Optical Character Recognition For Cursive Handwriting”, IEEE Trans Pattern Analysis and Machine Intelligence Vol.24, pp.801-813, 2002

[5] U. Pal, A. Belaïd and C. Choisy. "Water Reservoir Based Approach for Touching Numeral Segmentation". Group READ, LORIA, Campus Scientifique B. P. 23954506 Vandoeuvre-Les-Nancy, France.

[6] Y K Chen and J F Wang, " Segmentation of handwritten connected numerals string using background and foreground analysis," Proc. 15th ICPR, pp 598-601, 2000. 
Table 1. Table captions should be placed above the table

\begin{tabular}{|c|c|c|c|c|c|c|c|c|c|c|c|}
\hline & $\mathbf{0}$ & $\mathbf{1}$ & $\mathbf{2}$ & $\mathbf{3}$ & $\mathbf{4}$ & $\mathbf{5}$ & $\mathbf{6}$ & $\mathbf{7}$ & $\mathbf{8}$ & $\mathbf{9}$ & None \\
\hline $\mathbf{0}$ & $\mathbf{9 5 . 2 3}$ & 0 & 0 & 0 & 0 & 0 & 0 & 0 & 1.19 & 0 & 3.57 \\
\hline $\mathbf{1}$ & 0 & $\mathbf{8 4}$ & 4 & 0 & 0 & 4 & 0 & 0 & 0 & 0 & 8 \\
\hline $\mathbf{2}$ & 0 & 5.26 & $\mathbf{9 4 . 7 3}$ & 0 & 0 & 0 & 0 & 0 & 0 & 0 & 0 \\
\hline $\mathbf{3}$ & 0 & 0 & 0 & $\mathbf{6 6 . 6 7}$ & 8.33 & 0 & 0 & 0 & 0 & 0 & 25 \\
\hline $\mathbf{4}$ & 0 & 0 & 0 & 0 & $\mathbf{8 0}$ & 6.66 & 6.66 & 0 & 0 & 0 & 6.66 \\
\hline $\mathbf{5}$ & 0 & 2.63 & 0 & 0 & 0 & $\mathbf{8 9 . 4 7}$ & 2.63 & 0 & 0 & 0 & 5.26 \\
\hline $\mathbf{6}$ & 0 & 0 & 0 & 0 & 0 & 0 & $\mathbf{9 4 . 7 3}$ & 0 & 0 & 0 & 5.26 \\
\hline $\mathbf{7}$ & 0 & 0 & 0 & 0 & 5.88 & 5.88 & 0 & $\mathbf{8 2 . 3 5}$ & 0 & 0 & 5.88 \\
\hline $\mathbf{8}$ & 0 & 0 & 0 & 0 & 0 & 0 & 0 & 0 & $\mathbf{8 8 . 8 9}$ & 0 & 11.11 \\
\hline $\mathbf{9}$ & 0 & 0 & 0 & 0 & 0 & 0 & 0 & 0 & 0 & $\mathbf{8 7 . 5}$ & 12.5 \\
\hline
\end{tabular}

